\title{
A Theoretical Model for Autism
}

\author{
LUÍS ALFREDO VIDAL DE CARVALHO ${ }^{\mathrm{a} *}$, NÍVEA DE CARVALHO FERREIRA ${ }^{\mathrm{a}}$ and ADRIANA FISZMAN ${ }^{\mathrm{b}}$ \\ ${ }^{a}$ COPPE/UFRI, Program of Systems Engineering and Computer Science, P.O. Box 68511, Rio de Janeiro 21945-970, RJ, Brazil; \\ ${ }^{\mathrm{b}}$ IPUB/UFRJ, Institute of Psychiatry, Au Venceslau Bras, Rio de Janeiro, Brazil
}

(Received 31 March 2000)

\begin{abstract}
Autism is a mental disorder characterized by deficits in socialization, communication, and imagination. Along with the deficits, autistic children may show savant skills ("islets of ability") of unknown origin that puzzles their families and the psychologists. Comorbidity with epilepsy and mental retardation has brought the researchers' attention to neurobiological and cognitive theories of the syndrome. The present article proposes a neurobiological model for the autism based on the fundamental biological process of neuronal competition. A neural network capable of defining neural maps-synaptic projections preserving neighborhoods between two neural tissues-simulates the process of neurodevelopment. Experiments were performed reducing the level of neural growth factor released by the neurons, leading to illdeveloped maps and suggesting the cause of the aberrant neurogenesis present in autism. The computer simulations hint that brain regions responsible for the formation of higher level representations are impaired in autistic patients. The lack of this integrated representation of the world would result in the peculiar cognitive deficits of socialization, communication, and imagination and could also explain some "islets of abilities", like excellent memory for raw data and stimuli discrimination. The neuronal model is based on plausible biological findings and on recently developed cognitive theories of autism. Close relations are established between the computational properties of the neural network model and the cognitive theory of autism denominated "weak central coherence", bringing some insight to the understanding of the disorder.
\end{abstract}

Keywords: Autism; Weak central coherence; Cortical maps; Plasticity; Neurodevelopment; Neural networks

\section{INTRODUCTION}

Autism is a psychiatric disorder that was first described by Leo Kanner in 1943. He observed four fundamental characteristics in the disease: extreme autistic aloneness, failure to use language in a communicative fashion, obsessive desire for the maintenance of sameness, and a tendency to repeat the same action in a ritualized manner [27]. Concurrently, though independently, Asperger [3]

*Corresponding author. E-mail: okay@lamwaiting.com 
also used the term autism to refer to the core features of the disorder.

The increased perception of infantile autism as a distinct nosological entity led to a great deal of research and clinical speculation and to the development of the first operationally precise diagnostic criteriat in 1978 [40,43]. Almost at the same time, other authors [49] defined what they thought to be the core features of the disease, namely impairments in socialization, communication, and imagination. These features form a triad, since they co-occur and persist during the development of the child even though their outward manifestation is subject to change.

The notion of the core features triad represents a much wider autistic spectrum than the Kanner autism and form the basis for the current diagnosis, as set out in the 4th edition of Diagnostic and Statistical Manual of Mental Disorders, DSM-IV [2]. The DSM-IV diagnostic criteria for Infantile Autism parallel the International Classification of Diseases 10th Edition (CID-10) diagnostic criteria for Childhood Autism [48], both listed under the general heading of pervasive developmental disorders (PDD). Both, DSM-IV and CID-10 criteria, include reliable items consonant with clinical judgment and can be summarized as qualitative impairment in social communication and restricted and repetitive patterns of behavior and interests. These features must be evident before three years of age, although diagnosis is often made much later.

Along with the lack of gesture or verbal language, poor eye contact, echolalia, perseveration, and low generalization capacity, some autistic patients (approximately one case in ten) display islets of exceptional abilities (the so-called "savant skills") against a background of widespread cognitive impairment, such as calendar and prime number calculation, counting, memory, drawing, and music. The higher capacity in performing these tasks is not learned but it just emerges spontaneously without explanation so far and it does appear to be specific. Other types of mental handicap or developmental disorder do not share this profile [20].
According to the strict criteria applied by Kanner, autism is a rare disease and appears in four of every 10,000 births. With the somewhat wider criteria used in current diagnostic practice, the incidence is much higher: one or two in 1000 births, about the same as Down's syndrome [13]. The male/female ratio is between $2: 1$ and $2.9: 1$, albeit the preponderance is greater in the narrowly defined Kanner autism, with sex ratios ranging from $2.6: 1$ to $5.7: 1$.

Mental retardation is one of the most strongly associated, but not the defining, features of autism. About three-quarters of the autistic population have IQs in the retarded range. Regardless of the low overall IQ, the intellectual abilities of the autistic children show marked variations, ups and downs commonly called "spiky profile", that have been intriguing the researchers until now.

During the two decades following Kanner's original paper, researchers tried to discover an organic cause to the disease but, due to the absence of clear neurological evidences, their effort was in vain. Psychodynamic theories for autism appeared in the seventies, blaming parents for the symptoms of their autistic children and resulting in a plethora of inappropriate and ineffective therapies.

Two broad classes of medical conditions have been connected with autism: those with genetic basis (such as tuberous sclerosis or fragile $\mathrm{X}$ syndrome) and those traceable to pre, peri, or post-natal insults or infections (such as rubella embryopathy or herpes encephalitis). Genetic conditions may represent familial traits or spontaneous mutations. All twin studies support the existence of a strong genetic contribution to autism. The principal hypothesis for the mode of transmission is that the diathesis is a poligenical, multifactorial tract, with a number of different genes contributing additively to a dimension of vulnerability [11].

Neuropathological studies have shown increased neuronal density and smaller neurons in the hippocampus, entorhinal cortex, amygdala, and cingulate cortex. Neuronal loss was observed in Broca's area and also in the cerebellar cortex [5]. Reduced dendritic branching was also detected in CAl and CA4 hippocampal pyramidal cells [37]. Magnetic resonance imaging tended to reveal 
abnormalities in the forebrain and hindbrain, consistent with some of the neuropathological findings described above, mainly related to aberrant development [8]. It seems that there is much variation in the neuropathology of the autism, but abnormal wiring patterns, possibly due to problems in neuronal migration during development [36], truncation of normal neuronal growth [6] or aberrant neurogenesis [8] would result in increase and reduction of neurons and synapses in different brain areas.

Indeed, a neuronal model was proposed some years ago exploring the findings of increased or reduced neurons and synapses in autistic brains [7]. Computer simulations of that neural network have shown that good discrimination of stimuli is accomplished when there are too many neurons in one specific layer of the neural network, while generalization properties are obtained only when few neurons are present in the referred layer. The author associated these wellknown results of the theory of artificial neural networks [42] with the deficits of concept acquisition and generalization and also with the "islet of ability" in stimuli discrimination observed in autism.

The question of how a neural circuit with too many or few neurons arises from the process of neurodevelopment was not addressed by the Cohen's model. In the present work, a more realistic model is proposed. Based on the fundamental biological process of neuronal competition, a neural network capable of organizing its structure as a function of the stimuli received will, as in a process of neurodevelopment, recruit more or less neurons and make more or less synapses, bringing insight to the cause of the aberrant neurogenesis of autism. In other words, this work shows how excessive neuronal inhibition in some neurodevelopmental phase can result in aberrant neural circuits with computational abilities similar to those observed in autism. The computer simulations suggest that brain regions responsible for the formation of higher level representations are impaired in autistic patients. The lack of this integrated representation of the world would lead to the peculiar cognitive deficits of socialization, communication, and imagination but could also explain some "islets of abilities" like excellent memory and stimuli discrimi- nation. The neuronal model is based on plausible biological findings and on recently developed cognitive theories of autism.

\section{THE COGNITIVE THEORIES OF AUTISM}

The generality of the neuropathological findings, allied to the failure of the psychodynamic theories, gave impetus to cognitive scientists in their search for theoretical bridges between autistic brain and behavior. One of the most general cognitive theories for the autism is based on the social impairment imposed by the disease. According to this theory, three core areas of social interaction (imitation, joint attention and interpersonal relatedness) are impaired in an early developmental phase, resulting in serious limitations later. The basis of social understanding lies in the child's comprehension that the others are like her, and then capable of sharing the same emotional and cognitive impressions about any object. Problems of interpersonal relatedness since early developmental phases of the child, would, later, cause serious communicative and social deficits. Studies in congenitally blind children with autistic behavior may corroborate this hypothesis [23]. Joint attention-the process of sharing the same focus of attention alternating eye gaze and pointing between an object and another person - is also lacking in autistic children. The failure to use eye gaze for communication, the lack of interpretation of one's emotional expressions, and the incapacity of understanding one's reactions are characteristics of autistic children that seem to be related to poor joint attention [28]. Finally, imitation is the third process that autistic children do not develop. A normal child imitates faces trying to express or feel the same emotion that the face seems to show. Imitation of others is the basic tutorial guide to social life [34].

Another important cognitive theory of autism hypothesizes that autistic children cannot represent the mental state of themselves and others and, consequently, cannot understand or predict one's behavior. An autistic child can transmit a message to someone and, after that, cannot differentiate who 
knows from who ignores the message. As a consequence of this lack of a theory of mind of the other, autistic children are impaired in their understanding of mental states. They cannot pretend play because they do not have the intuitive ability to distinguish physical states (real situation) from pretended mind states [4]. Although deficits in socialization, communication and imagination define the core of the autistic syndromes, a repetitive, restricted and stereotyped behavior is also present. Due to the similarity of this pattern to patients with frontal lobe damage, another cognitive theory for autism arises: the executive function theory. We understand by executive functions the cognitive processes like the ability to disengage from a context, the use of feedback from the environment, shifting attention, planning, and the inhibition of inappropriate action. The hypothesis of the executive function theory of autism is that some developmental disorders cause disturbances in neuronal circuits of the frontal lobe or hippocampus or yet the basal ganglia [47]. The theory is weakened by the fact that some autistic children have "islets of abilities" in tasks that demand intact frontal lobe functions [9].

All the theories of autism already shown are capable of explaining cognitive deficits but none of them touch the question of the "islets of ability" or the spiky profile in the neuropsychological assessment. The recently established weak central coherence theory states that autistic children have a peak of performance in activities that do not require integration but, on the contrary, segmentation of information $[14,15]$. In accordance to this theory, autistic brains would have a cognitive style that favors segmentation due to the limitation or impossibility of a more holistic information processing. Experiments on language, memory, and perception [16] have shown that autistic children are not strongly disadvantaged, in relation to normal children, when processing meaningless verbal material. Non-autistic children have a tendency to search for associations and other types of integration of the processed information, having a low performance when unconnected or meaningless material is presented in verbal assessment. Autistic patients also achieve good performance in visuo-spatial tasks where image segmentation is necessary [44]. Findings of less coherence in narratives and superior visuo-spatial skills in non-autistic relatives of autistic children suggest that the lack of central coherence in these patients is genetically transmitted [32]. It is worth noting that adults with autism but who can pass the theory of mind tests show cognitive patterns of weak central coherence [19]. This result hints the possibility that weak central coherence is an independent and additional feature of autism, with no obvious relation, in principle, to theory of mind deficits.

\section{NEURAL COOPERATION AND COMPETITION}

In the middle of the 19th century the scientists Helmholtz and Mach studied many phenomena of the visual perception in humans. Particularly, they were interested in optical illusions like the fact that edges or contours between light and dark parts of an image tended to be enhanced in relation to the light and dark interior of the image. They explained the illusion hypothesizing that in the human retina the cells are excited by light that converges to a central region and are inhibited by the light that projects to the surrounding areas. Almost a century later, experimental results showed that the eye of the crab called Limulus [21] and some vertebrates [31] have an structure, then called on-center/off-surround, in which a neuron is in cooperation, through excitatory synapses, with the neurons in the immediate neighborhood while it is in competition with the neurons which lay outside these surroundings. There is experimental evidence supporting that the same mechanism is also present in the mammalian central and peripheral nervous system. It seems that pyramidal cortical cells are connected in this oncenter/off-surround way [46]. Other areas in the brain, like the hippocampus [1] and the cerebellum [10] have the referred hardwired structure.

Competition and cooperation are found not only statically hardwired but also as part of many neuronal dynamical processes. As a matter of fact, competition 
is essential to the neurodevelopment where neurons compete for certain chemicals. In synaptogenesis, for example, the substances generically called neural growth factors are released by stimulated neurons and, spreading through diffusion, reach the neighboring cells, promoting synaptic growth. Cells that receive neural growth factors make synapses and live, while the cells that have no contact with these substances die $[25,26]$. A neuron that releases neural growth factor guides the process of synaptic formation in its tridimensional neighborhood, becoming a center of synaptic convergence. When some neighboring neurons release different neural growth factors in different amounts, many synaptic convergence centers are generated and a competition is established between them by the synapses of their surroundings. It seems that at least two processes participate in the dynamics of synaptic formation: pre-synaptic neurons competing for neural growth factors to survive and post-synaptic neurons that release neural growth factors competing for synapses that will keep them alive with stimuli. It is worth noting that, as a single neuron is capable of receiving and releasing neural growth factors at the same time, the two competition processes described above effectively occur in every neuron and, consequently, a signaling network is established to control the development of neural circuits. Remembering that all this competition is started and controlled by environmental stimulation, it is possible to have a glimpse to the way the environment records or represents itself in the brain.

The competition processes described above are essential to the formation of some neuronal organizations called maps. A neural map is a biological circuit composed of two sets of neurons, called domain and image, in such a way that similar patterns of activation of the domain are projected to neighboring neurons in the image. In other words, a neural map is a projection that transfers similarities at the domain to spatial relationships at the image. Studies of the visual [24], somatosensory [35], and associative [17] cortices showed that small regions of those tissues respond to similar stimuli. Indeed, stimuli like position, orientation, color, spatial

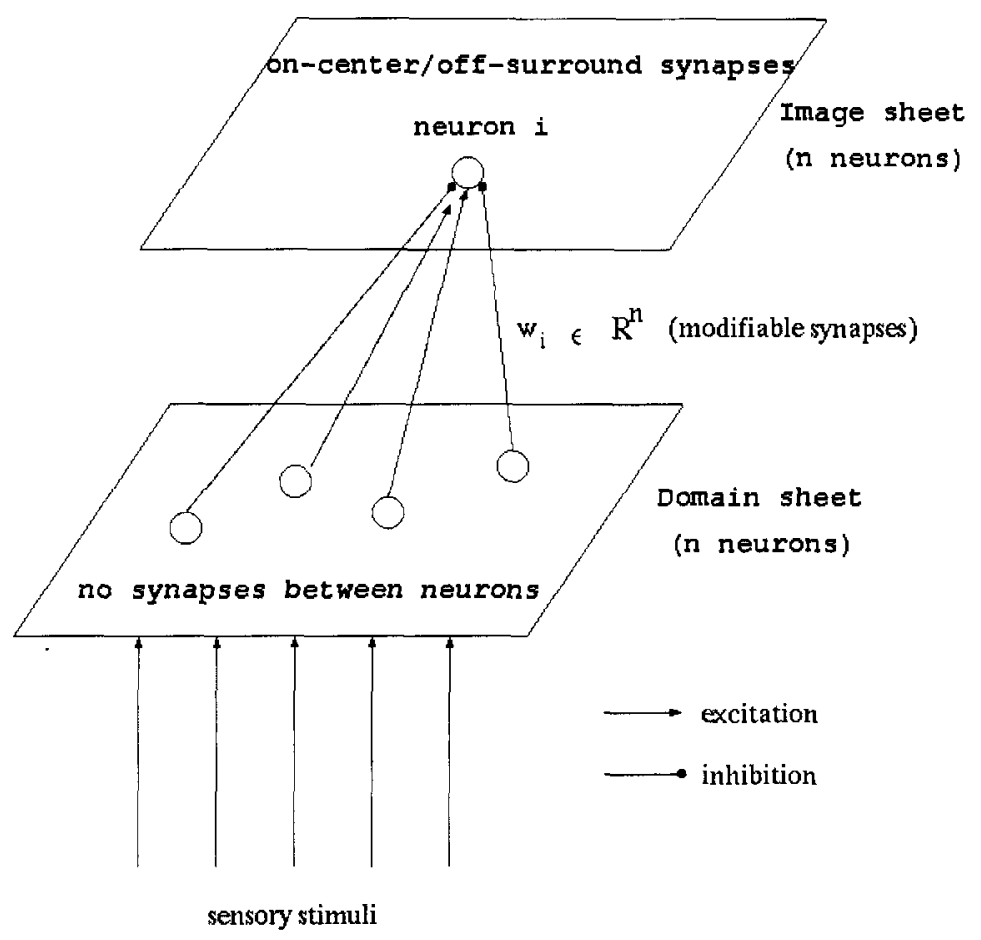

FIGURE 1 A self-organizing map with two bidimensional sheets of neurons. 
frequency, auditory frequency, and also meanings $[38,39,41,45]$ are capable of being represented in neuronal circuits as maps. Maps have puzzled neuroscientists in the last decades, mainly the question of how do they arise from the simple oncenter/off-surround wiring pattern. Computational theories gave some important insights to the problem, since some cortical maps are artificially developed from simple governing rules of synaptic plasticity in computer simulation models [33]. The most general of these models is called the Self-Organizing Map [30] in which two sheets of neuronal tissue with $n$ neurons each, corresponding to the domain and the image, are initially randomly connected in a way that every neuron $i$ at the image receives synaptic projections $w_{i} \in R^{n}$ from every neuron at the domain (Fig. 1). Neurons at the domain do not form synapses among themselves and receive "sensory" inputs (stimuli), while neurons at the image make synapses following the on-center/off-surrounding paradigm, i.e. shortrange excitation or cooperation and long-range inhibition or competition (Fig. 2).

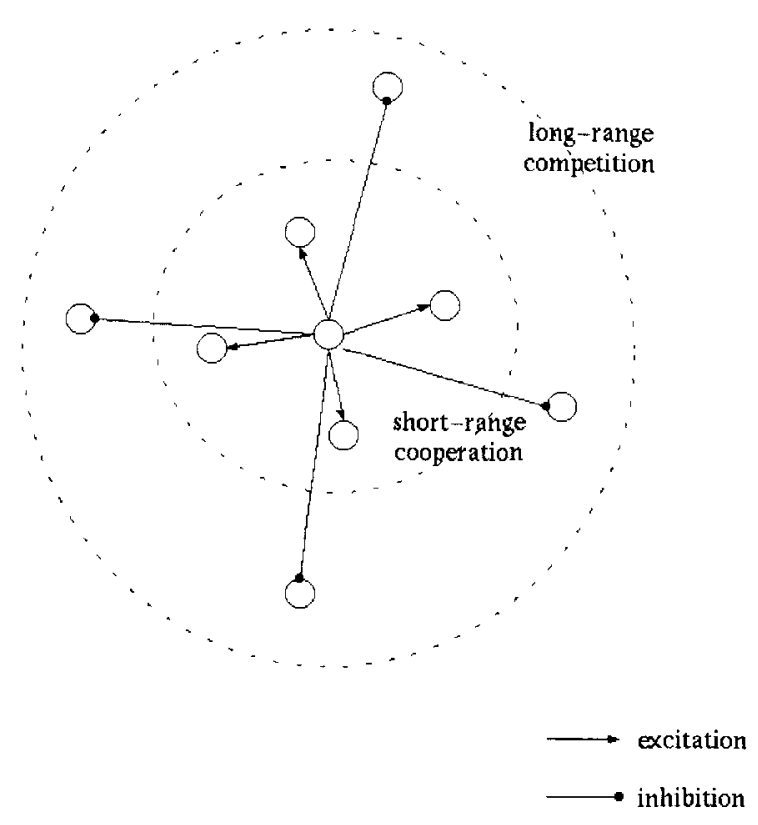

FIGURE 2 The on-center/off-surrounding synaptical pattern of the image neuronal sheet.
The on-center/off-surround synapses do not change during the development of the map, while the synapses between the domain and the image are modified along the process of map formation. Indeed, every time the neural network is in contact with a stimulus $x_{k} \in R^{n}, k=1,2, \ldots$ in its domain, there will be only one excited neuron $i^{*}$ at the image because the short-range cooperation and long-range competition makes the more excited neuron inhibit the others. The position $r^{*}$ of this winner neuron at the image determines how much synapses will be modified. Synapses from neurons closer to the winner will be strongly changed in such a way that these neurons will be more intensely excited by the stimulus $x_{k}$ in a next time. Synapses from neurons distant from the winner will be weakly changed or not changed at all, depending on the dispersion $\sigma$ of the neighborhood function $\phi\left(r_{i}, r^{*}\right)$, where $r_{i} \in R^{n}$ gives the position of neuron $i$ at the image sheet. By this process, every neuron in the image will be more easily excited by the stimulus $x_{k}$ (synaptic facilitation) in the future. The development of the map is due to the fact that the amount of synaptic facilitation is proportional to the distance from the winner neuron. The process of synaptic modification $\Delta w_{i}^{l}$ for each neuron $i$ is repeated for every learning step $l$ where the stimulus $x_{k} \in R^{n}, k=1,2, \ldots$ is presented to the neural network, and is given by

$$
\Delta w_{i}^{l}=\rho(l) \phi\left(r_{i}, r^{*}\right)\left(x_{k}-w_{i}\right)
$$

where $\rho(l)$ is the learning rate defined by

$$
\rho(l)=\rho_{0} \beta^{(l-1)} ; \quad 0<\beta<1, \quad l=1,2, \ldots
$$

The learning rate begins with the value $\rho_{0}$ and decreases with the learning step $l$ with a rate $\beta$.

The neighborhood symmetric function $\phi\left(r_{i}, r^{*}\right)$ takes the form of a gaussian function (Fig. 3) defined by

$$
\phi\left(r_{i}, r^{*}\right)=\exp \left\{-\left(\left\|r_{i}-r^{*}\right\|^{2} / 2 \sigma(l)^{2}\right) .\right.
$$

The initial dispersion of the gaussian, $\sigma_{0}$, is high, representing that all the neurons in the image are considered neighbors. This allows the modification of 
the randomness of the initial synapses to a more organized pattern where neighborhood is of capital importance. Every time step $l$ that another stimulus is presented to the neural network domain, the neighborhood shrinks a bit, gradually giving to the map a local organization. The dispersion $\sigma(l)$ at each learning step is given by

$$
\sigma(l)=\sigma_{0} \alpha^{(l-1)} ; \quad 0<\alpha<1, \quad l=1,2, \ldots
$$

where $\alpha$ is a decrement rate.

The way the learning rate decreases and the neighborhood shrinks is fundamental to the map development. A faster decrement in the learning rate does not give enough time to the synapses to change, and so the randomness of the initial synaptic pattern is consolidated at the end of the process. When neighborhoods shrink rapidly, the level of neuronal cooperation necessary to produce maps is not present and neighborhood relationships are ill defined at the end of the simulation. Indeed, the neighborhood function may be likened to the steady-state concentration profile of neural growth factor in the neural tissue. When the dynamical equilibrium between neural growth factor release and metabolization is accomplished in every region of the tissue, due to the diffusion process, a concentration profile that asymptotically decreases with radial distance is attained (see Fig. 3). The parameter $\sigma_{0}$ represents the amount of neural growth factor released by the neurons at the beginning of the neurodevelopment process.

An efficient way of assessing the effectiveness of a neurodevelopment process is to measure the total synaptic change occurred. It is observed that largescale changes in the synaptic pattern get along with successful neural circuits $[25,26]$. When synapses are not changed (weakened or strengthened) the neurodevelopment process has not succeeded and the final map is similar to the initial one. At each learning step $l$ of the self-organizing algorithm, a winner neuron determines the intensity of the synaptic changes for every neuron $i$ by Eq. (1). Summing the amount of synaptic change $\Delta w_{i}^{l}$ for all neurons at every learning step $l$ gives us the total synaptic change $S$ of the selforganizing process:

$$
S \equiv \Sigma_{l} \Sigma_{n}\left\|\Delta w_{i}^{l}\right\|
$$

In the next section, some simulation experiments show how abnormal neural maps may develop from self-organizing processes, resulting in neural networks with computational abilities capable of explaining some cognitive characteristics of autistic children.

\section{SIMULATION RESULTS}

A self-organizing map to represent the synaptic development between two cortical bidimensional sheets was implemented for computer simulation. A lower level cortical sheet (here called domain) with 400 neurons receives input stimuli from sensory areas and projects its output to the higher level cortical sheet (here called image) also with 400 neurons (see Fig. 1). A thousand input stimuli were randomly generated. The range of the random number generator was changed four times in a way of producing four different sets of 250 stimuli each. Indeed, these sets define clusters of relatively similar input patterns, as can be seen in Fig. 4, where the stimuli of each cluster

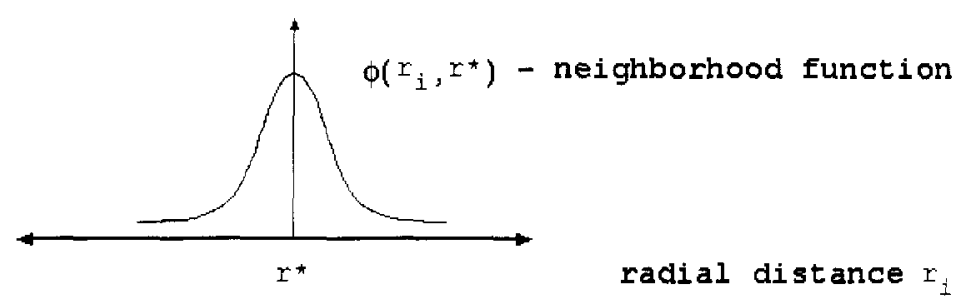

FIGURE 3 The neighborhood function representing the steady-state concentration of a neural growth factor. 
are represented over a bidimensional sheet of cortical neurons by the same geometric marker.

Initially, the synaptic weights are defined at random. As a consequence, the stimuli presented to the domain cortical sheet (shown in Fig. 4) are projected to random positions at the image cortical sheet, as represented in Fig. 5. The similarities between the stimuli are not preserved at the higher level cortical sheet, or in other words, on map-like representation exists. In all experiments that follow, the domain cortical sheet was excited 25 times with the four clusters of 250 stimuli in a random manner. The parameters $\rho_{0}, \alpha$ and $\beta$ are kept constant in 0.8 , 0.99 , and 0.99 , respectively, for all simulations.

As $\sigma_{0}$ represents the amount of neural growth factor released by the neurons of the image cortical sheet at the beginning of the neurodevelopment process, in a first simulation this parameter was set to a high value $\left(\sigma_{0}=4.0\right)$, sufficient to the correct development of the map. After the execution of the self-organizing algorithm, the four clusters of stimuli presented to the domain cortical sheet (Fig. 4) were mapped into four different and well-defined regions, as can be seen in Fig. 6. During the simulation of the neurodevelopment process, the synapses between the two cortical sheets evolved in time with a total synaptic change $S$ equal to 11.3 , transforming the initially disorganized map (Fig. 5) into a clearly organized one (Fig. 6). Note that the 250 stimuli of each cluster were mapped into only 100 neurons (on average) at the image sheet. In fact, any other stimulus never before presented to the neural network, but similar to one of the 250 stimuli of a cluster, will be mapped directly into the region of the image cortical sheet responsible for the representation of that cluster. Imagining that during one's lifetime a multitude of similar stimuli are presented to one's brain, it is easy to conclude that cortical maps are intelligent structures capable of representing infinitely many instances or variations of a stimulus in a constant, well-defined and small region of the cortex. In other words, neural maps are capable of, based on a few examples or instances, create and hardwire (or represent) a whole category in the cortex. One obvious advantage of this capacity is the economy incurred: would every stimulus be represented by one specific neuron, billions of these cells should not be enough for a lifetime.

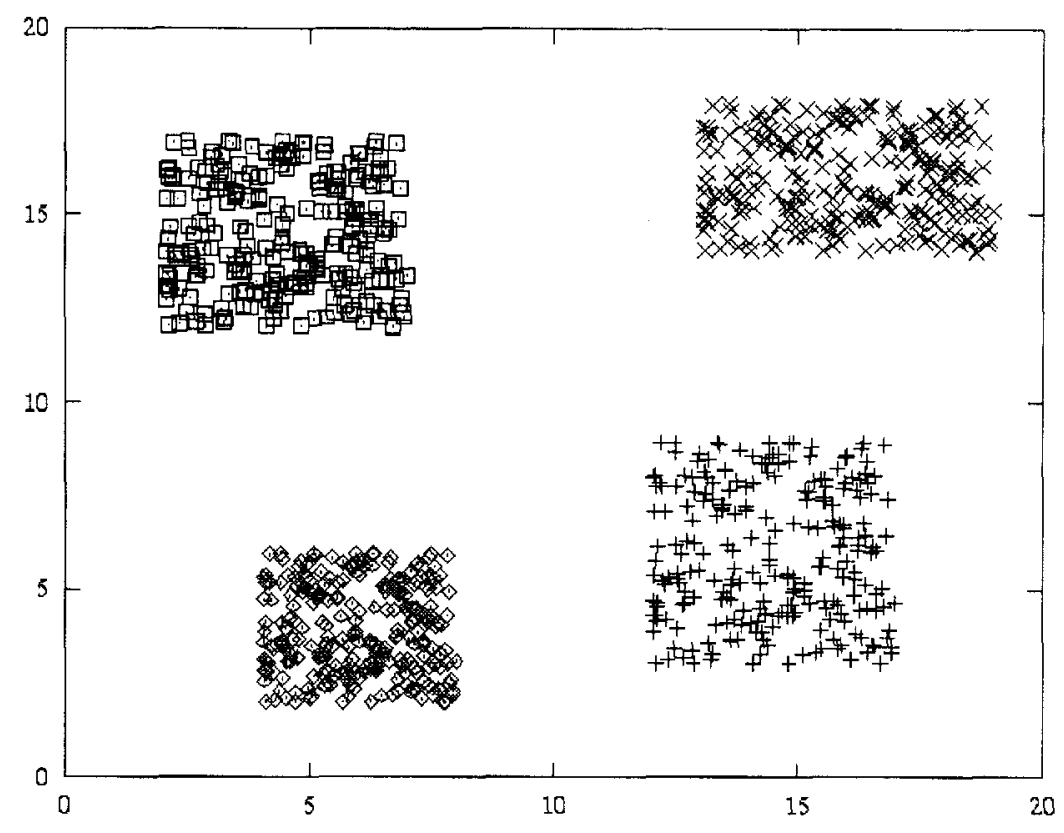

FIGURE 4 A thousand stimuli divided into four clusters of 250 stimuli each. 


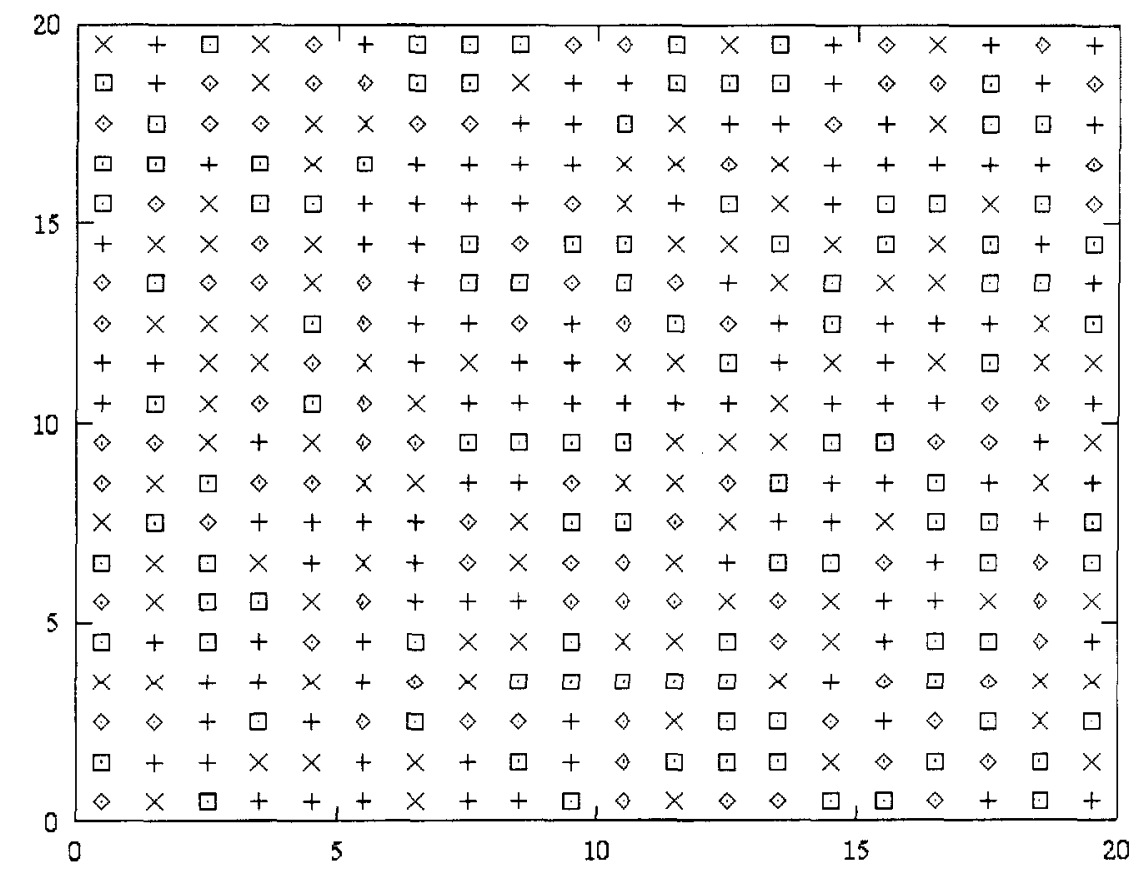

FIGURE 5 Due to the random character of the initial synaptic pattern, the stimuli clusters are projected to the image sheet in a disorganized way.

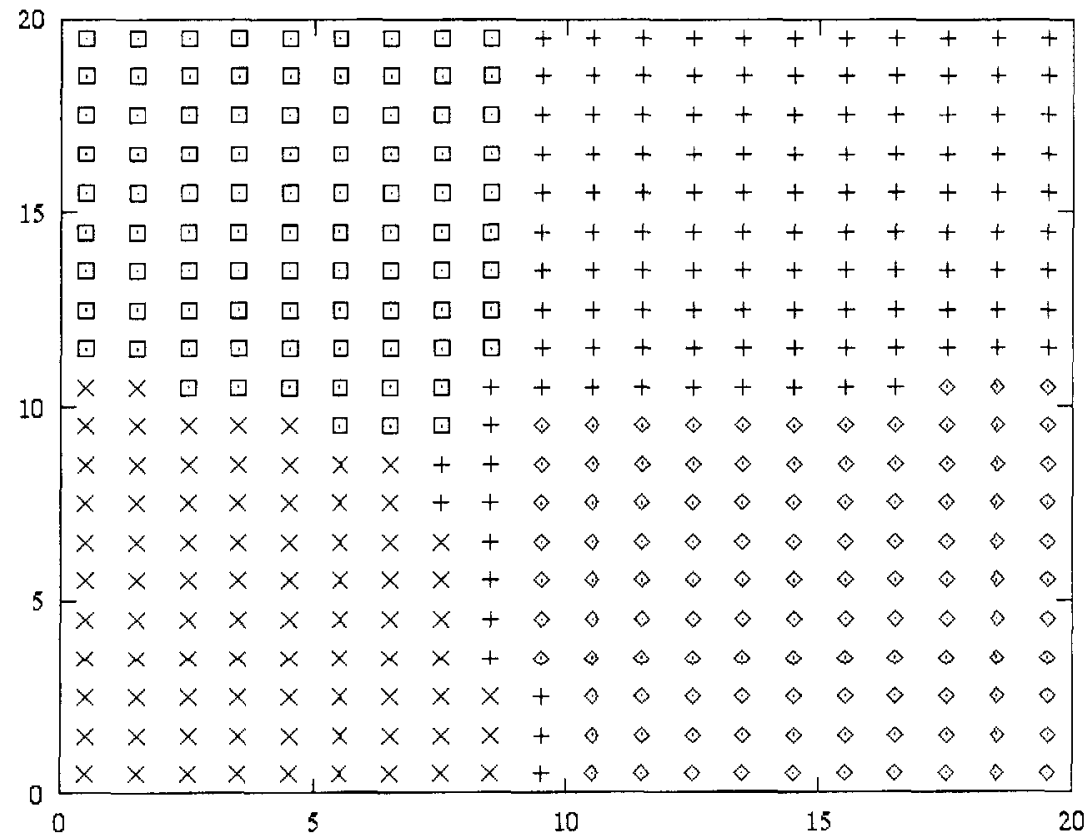

FIGURE 6 A well-developed cortical map generated with sufficient neural growth factor. 
The construction of these maps is possible just due to the fact that the cortical neurons and their synaptic plasticity governing rules build a neurocomputational circuit capable of extracting from the stimuli their commonalties. The cortical map considers the common features as the principal components (or the central coherence) of the stimulus, while the uncommon characteristics that would hinder the process of categorization are disregarded. In other words, cortical maps are possible because their circuits generalize the stimuli, i.e. they consider the general (common or central) features as the only ones that matter, while the specificities (details) of each stimulus are not computed.

A normal brain, charged with well-developed maps, is always, and naturally, searching for the central coherence of every new stimulus that reaches the cortex. Finding the central coherence is an extremely important cognitive style of normal minds, since in a complex and ever changing environment it is a great deal to recognize a new situation as similar to an old one for which a good response is already known.
Once analyzed the computational properties and the cognitive implications of well-developed cortical maps, the next four similar simulations will show how ill-developed maps have characteristics that resemble autistic minds. To achieve this purpose, the parameter $\sigma_{0}$ will be decreased at each simulation, showing how the reduction of neural growth factor degrades the map and changes its computational abilities. Starting with $\sigma_{0}=3.5$ (a reduction of $12.5 \%$ in relation to $\sigma_{0}=4.0$ of the successful map), the selforganizing process departed from the same randomgenerated map (Fig. 5) and reached the final state shown in Fig. 7. Comparing the two maps (Figs. 6 and 7) we note that the last one was completely reshaped. The regions responsible for the clusters of stimuli assumed different forms and relative dispositions at the image cortical sheet. Nevertheless, the computational, and consequently the cognitive, properties of the map are the same: categorization, generalization, central coherence or any other word that express common feature recognition. A closer look to this map (Fig. 7), however, allows us to observe that at the upper left corner of the image cortical sheet there are

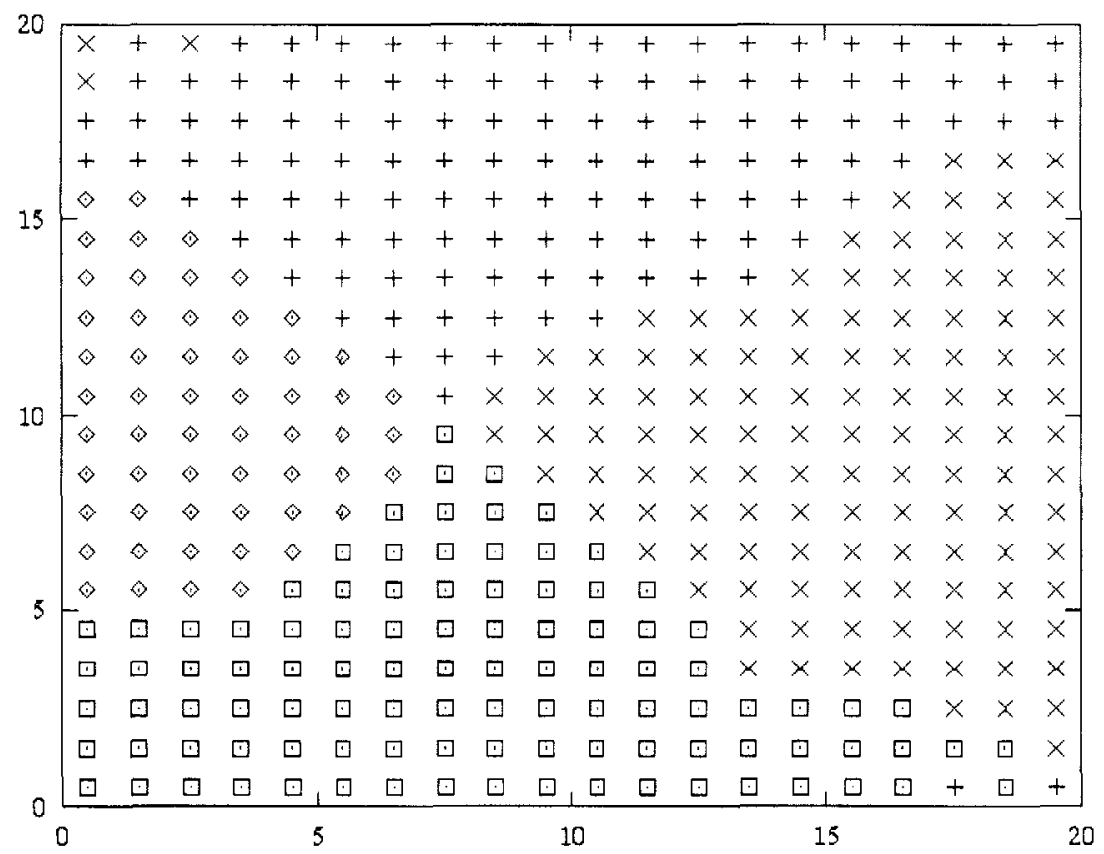

FIGURE 7 A cortical map developed with a reduced amount of neural growth factor $\left(\sigma_{0}=3.5\right)$. Some stimuli are misclassified. 
three stimuli of the " $\mathrm{X}$ " cluster misclassified into the "+" cluster region. Also, some misclassified stimuli are present in the lower right corner of the same map.

A more drastic reduction in the amount of neural growth factor offered $\left(\sigma_{0}=2.5\right)$, i.e. $37.5 \%$ reduction in relation to the $\sigma_{0}=4.0$ of the successful map, leads to the formation of the map depicted in Fig. 8. Note that the existence of a big region for each cluster of stimuli parallels the emergence of a new, relatively smaller, region with stimuli of the same cluster. The number of stimuli of each cluster out of the main cluster region is so high that the concept formerly used of "misclassification" (Fig. 7) no longer makes sense. As a matter of fact, the clusters seem to be split in half. Form the computational point of view, the selforganizing process generated a map that extracts from the stimuli not only the commonalties but also some specificities. The specific features are capable of differentiating the stimuli, forcing the map to create another category for them because they are not so similar to be classified in a single cortical region. We may say that the reduction of neural growth factor seems to generate a more discriminative map. The former capacity of generalization, or central coherence determination, is weakened and a segmentation or discrimination property arises. Details, formerly disregarded by the map, are now considered, adding a new dimension to the problem of defining a measure of similarity between stimuli. Sets of stimuli formerly considered similar and projected to the same cortical area, now, due to the higher discriminative capacity of the map, are perceived as different enough to require a new cortical region to their representation. The cognitive consequences of this emergent computational style is that a new situation that was easily recognized and processed as an old one, to which a response already existed, is now treated as a brand new event to which a response must be developed.

When the simulation is performed with a still smaller value of 1.5 for the parameter $\sigma_{0}$ (a reduction of $62.5 \%$ ), the discriminative character of the neural map is enhanced (Fig. 9). Every cluster of stimuli is now classified in many different categories or, equivalently, in distant cortical areas. A final and extreme reduction of neural growth factor $\left(\sigma_{0}=\right.$ 1.0) produces a completely segmented map, as seen

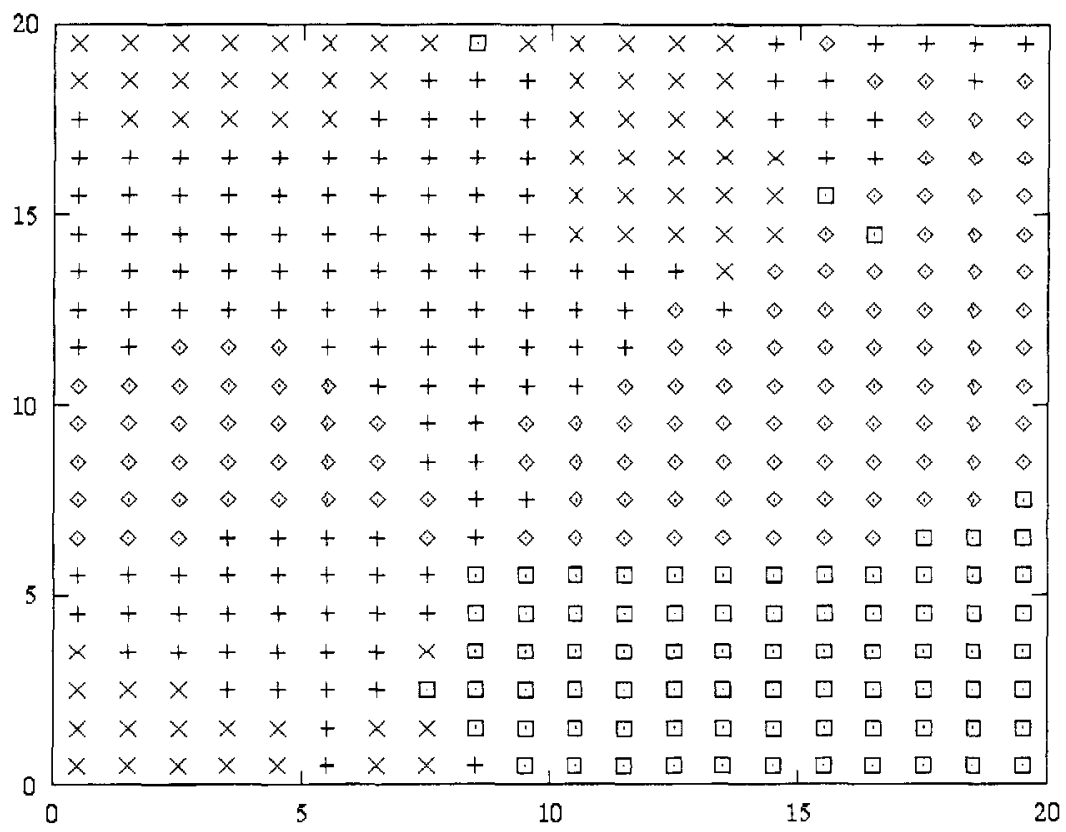

FIGURE 8 Another cortical map now developed with a more drastic reduction in the amount of neural growth factor $\left(\sigma_{0}=2.5\right)$, Some stimuli clusters are split in half. 
in Fig. 10. This last simulation shows us the "dual map" of the first simulation. Maps with high discriminative capacity and weak central coherence extraction (Fig. 10) represent the other side of the medal in relation to the maps with high generalization property and strong central coherence detection (Fig. 6). Between these two extremes there are a plethora of self-organizing maps, possibly resulting in different levels of the same neurological syndrome or, worse, nosologically different neurological syndromes with the same basic etiology. The curve depicted in Fig. 11 shows how the total synaptic change $S$ varies with the neural growth factor released at the start of the simulated neurodevelopment process. It is possible to conclude that weak central coherence extraction maps are consequence of low levels of neural growth factors. These maps are immature because their synapses could not change (low $S$ value) and were not submitted to the process of strengthening and consolidation or weakening and elimination [26]. Note that an excessive number of synapses is found in autistic brains [29], and this is in accordance to the low value of the total synaptic change $S$ found in the weak central coherence maps developed here.

The simulations performed until now allow us to extrapolate some result to the cognition and symptomatology of autism. To begin with, some authors noticed that "autistic children do not tend to integrate current experiences with previous impressions" [22] and also that "autistic children miss what is salient and pay attention to what is irrelevant" [18]. These two phrases pop out from our simulations every time a reduction of neural growth factor produces a neural map with less generalization (integration) capacity and higher discrimination (attention to details). Highly discriminative cortical maps will lead to a cognitive style where the stimuli are segmented and stored as a function of the details. As a consequence, cognition will be guided by the recognition of parts, possibly promoting a behavior characterized by excessive precision and inflexibility. For autistic children, a word must be pronounced always the

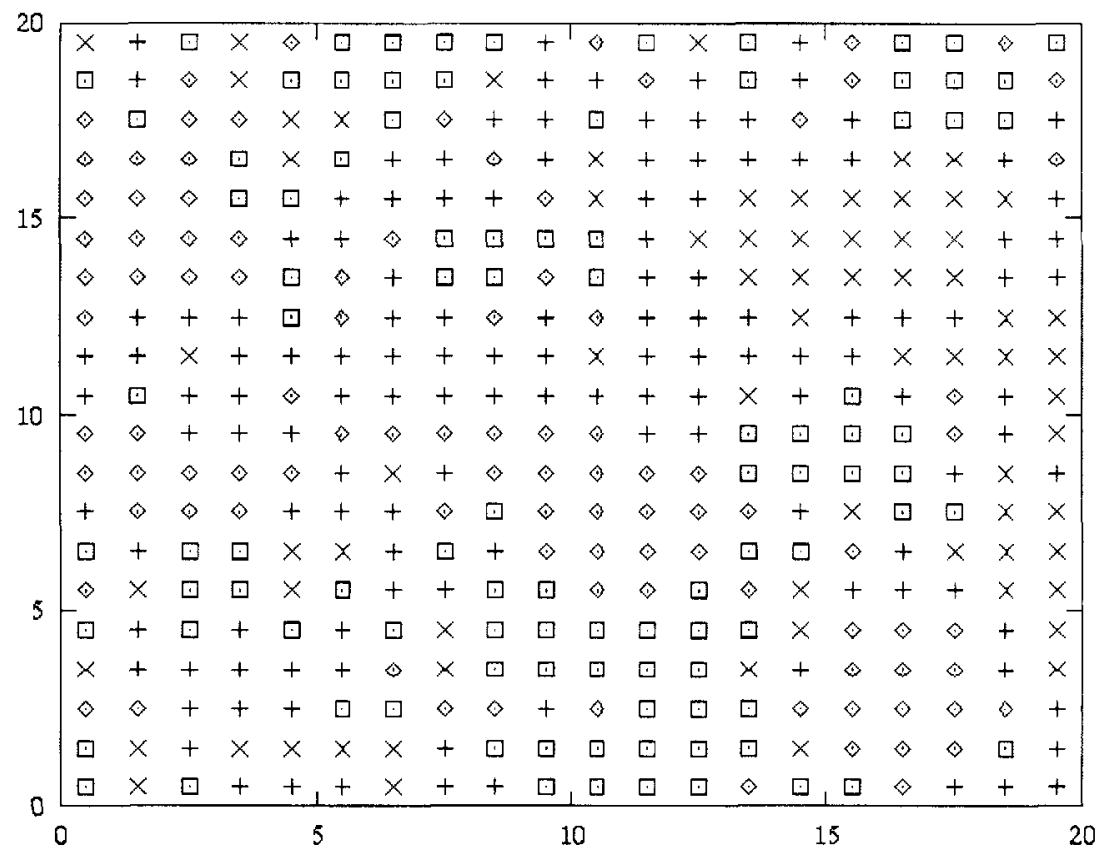

FIGURE 9 A smaller release of neural growth factor $\left(\sigma_{0}=1.5\right)$ generates a more discriminative map. The stimuli clusters are classified into many different categories. 


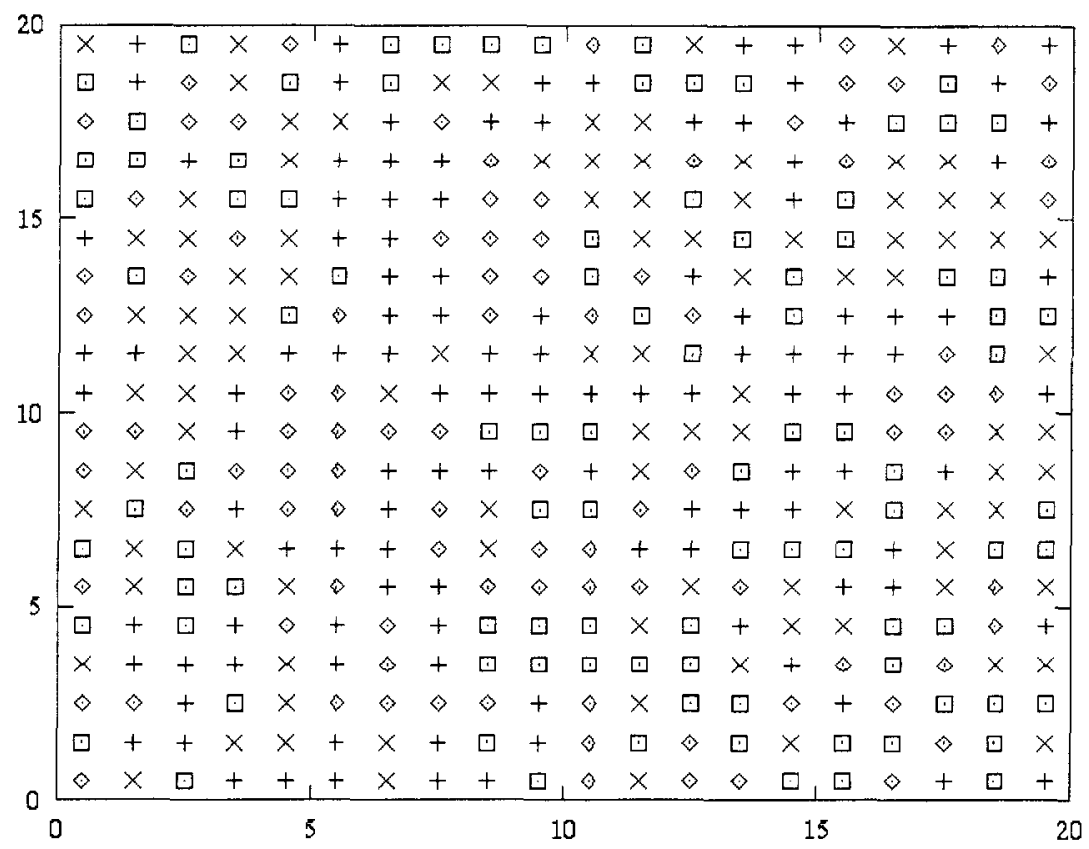

FIGURE 10 An extreme reduction of neural growth factor release $\left(\sigma_{0}=1.0\right)$ generates a completely segmented map.

same way or it will not be understood, the school bathroom is not a bathroom because the objects have a different spatial disposition, and the way back home must cross the same streets it is not the way back home.

Another cognitive consequence of highly discriminative maps is that information (stimulus) is not categorized but represented by segments or instances. The principle of economy is, then, broken and a huge amount of neurons will be necessary to represent concepts that normally would be mapped in just a small cortical region, as our simulations have shown. When large cortical regions are responsible for the representation of few detailed concepts, emerges a computational phenomenon called "combinatorial explosion" characterized by inefficient information processing due to insufficient hardware. The low IQ and the restricted interests in just a few subjectsremarkably well memorized, indeed-observed in autistic patients may be some result of the combinatorial explosion.

On the other hand, highly discriminative neuronal maps allow the automatic and fast segmentation of information and the representation of details. Possibly, this is the reason why autistic children have islets of ability in memory for raw data (names, phone numbers, calendar, realistic drawing, etc.), efficiently solve puzzles where parts of a context need to be quickly analyzed, and also have good sensory discrimination skills, for example to sounds and music [12].

Remembering that cortical processing is obtained from a series of hierarchical maps, where the input to a higher level map is the output of the immediately lower level map, it is possible to preview that cognitive problems will arise when the first map in the hierarchy classifies similar stimuli in different cortical regions. Perhaps, autistic minds cannot develop language skills because they do not have the proper higher level semantic maps [39] that depend on lower level well-formed maps. Also the social difficulties of the autism would be the result of the impossibility of recognizing emotional and facial expressions, tasks that need the interpretation of contexts and the integration information that occur in higher level maps. 


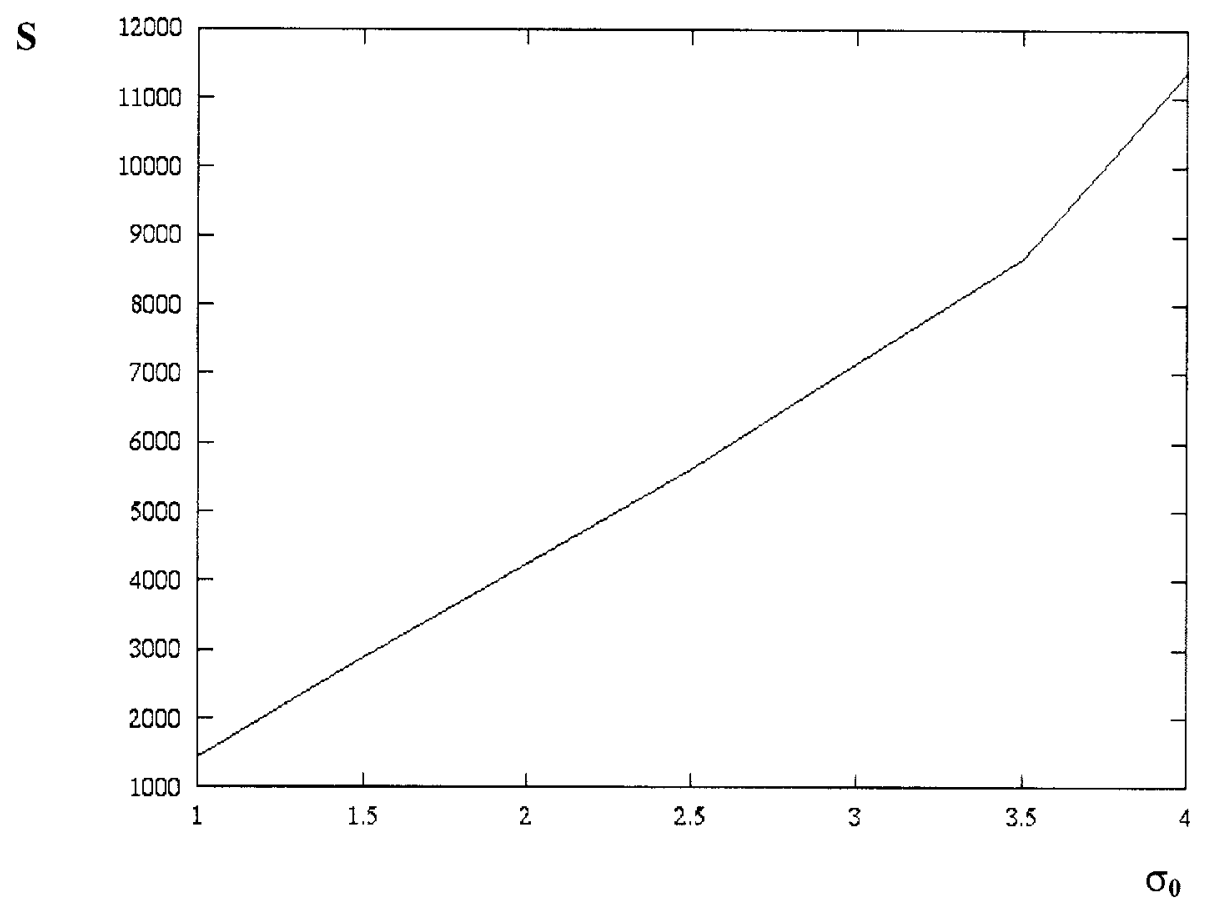

FIGURE 11 The total synaptic change as a function of the neural growth factor release.

\section{CONCLUSIONS}

Autism is marked by deficits in socialization, communication, and imagination but also by some islets of ability in operative skills involving memory and sensory discrimination. There are many cognitive theories for the disease but only one of them, the weak central coherence theory, is capable of explaining the referred islets of ability. After a detailed analysis of the concept of cortical map, a self-organizing algorithm to simulate the process of neurodevelopment of maps is presented. Some simulations performed showed how maps develop and what are their principal control parameters. One of these parameters was plausibly related to the important chemicals called neural growth factors and simulations proceeded to show that the amount of this substance released during the neurodevelopment process is capable of generating maps with a continuously varying generalization capacity. The computational properties of these maps were closely analyzed and related to the cognitive weak central coherence theory of autism. The lack of neural growth factor was shown to produce maps with low generalization property or weak central coherence extraction. Cognitive deficits and, the most important, cognitive skills (islets of ability) observed in autistic children were explained by analogies between the computational properties of the simulated maps and the weak central coherence theory. The analogies allow the authors to propose a neurocomputational model for the autism capable of linking biology to cognition in this disease. Albeit these analogies fit very well as a mean to understand autism from a neurobiological point of view, the existence of illdeveloped and highly discriminative cortical maps in autistic brains must be verified experimentally.

\section{Acknowledgements}

The authors would like to thank to Prof. João Ferreira da Silva Filho, director of the Institute of PsychiatryIPUB/UFRJ and Prof. Marlene Fiszman for all 
pertinent comments and valuable suggestions. We also thank the support given by the National Research Council $(\mathrm{CNPq})$ and the State Research Foundation (FAPERJ).

\section{References}

[1] Andersen, P., Gross, G.N., Lomo, T. and Sveen, O. (1969) The Interneuron (University of California Press, Los Angeles).

[2] APA (1994) Diagnostic and Statistical Manual of Mental Disorders, 4th ed. (American Psychiatric Association, Washington, DC).

[3] Asperger, H.D. (1944) "Autistischen psychopathen im kindesalter", Arch. Psychiat. Nervkrankh 117, 76-136.

[4] Baron-Cohen. S., Leslie, A.M. and Frith, U. (1985) "Does the autistic child have a theory of mind?", Cognition 21, 37-46.

[5] Bauman, M. (1991) "Microscopic neuroanatomic abnormalities in autism", Pediatrics 87(5), 791-796.

[6] Bauman, M. and Kemper, T.L. (1986) "Developmental cerebellar abnormalities: a consistent finding in a early infatile autism", Neurology, 36-190.

[7] Cohen, I.L. (1994) "An artificial neural network analogue of learning in autism", Biol. Psychiat. 36, 5-20.

[8] Courchesne, E., Press, G.A. and Yeung-Courchesne, R. (1993) "Parietal lobe abnormalities detected with MR in patients with infatile autism", Am. J. Roentgenol. 160, 387-393.

[9] Duncan, J. (1995) "Attension, intelligence and the frontal lobes", In: Gazzaniga, M.S., ed, The Cognitive Neurosciences (MIT Press, Cambridge, MA), pp 721 - 733 .

[10] Eccles, J.C., Ito, M. and Szentagothai, J. (1967) The Cerebellum as a Neuronal Machine (Springer, New York)

[11] Fein, D., Joy, S., Green, L.A. and Waterhouse, L. (1996) "Autism and pervasive developmental disorders", In: Fogel, B.S., Schiffer, R.B. and Rao, S.M., eds, Neuropsychiatry (Williams and Wilkins, Baltimore)

[12] Frith, U. (1989) Autism: Explaining the Enigma (Oxford University Press, Oxford, UK)

[13] Frith, U. (1993) "Autism: mysteries of the mind", Scient. Am., 92-98.

[14] Frith, U. (1996) "Cognitive explanations of autism", Acta Paediatr. Suppl. 416, 63-68.

[15] Frith, U. and Happe, F. (1994a) "Language and communication in the autistic disorders", Philos. Trans. R. Soc. London B. Biol. Sci. 346, 97-104.

[16] Frith, U. and Happe, F. (1994b) "Autism: beyond theory of mind", Cognition 50, 115-132.

[17] Goldman-Rakic, P.S. (1984) "Modular organization of the prefrontal cortex and regulation of behavior by representational memory", Trends Neurosci. 7, 419-429.

[18] Happe, F. (1991) The Autobiographical Writing of Three Asperger Syndrome Adults: Problems of Interpretations and Implications for Theory Autism and Asperger Syndrome, (Cambridge University Press, Cambridge, UK).

[19] Happe, F. (1994) "Wechsler IQ profile and theory of mind in autism: a research note", J. Child Psychol. Psychiat. 35, $1461-1471$.

[20] Happe, F. and Frith, U. (1996) "The neuropsychology of autism", Brian 119, 1377-1400.

[21] Hartline, H.K. and Ratliff, F. (1957) "Inhibitory interactions of receptor units in the eye of limulus", J. Gen. Physiol. 40, $351-376$
[22] Hermelin, B. (1978) Images and Language Autism: A Reappraisal of Concept and Treatment, (Plenum Press, New York)

[23] Hobson, P. (1993) Autism (Erlbaum, Sussex).

[24] Hubel, D.H. and Wiesel, T.N. (1965) "Receptive fields and functional architecture in two non-striate visual areas (18 and 19) of the cat", J. Neurophysiol. 28, 229-298.

[25] Kandel, E.R. (1991) "Cellular mechanisms of learning and the biological basis of individuality", In: Kandel, E.R., ed, Principles of Neuroscience (Appleton and Lange, Norwalk), pp 1009-1031.

[26] Kandel, K.R. and Jessel, T.M. (1991) "Early experience and the fine tuning of synaptic connections", In: Kandel, E.R., ed, Principles of Neuroscience (Appleton and Lange, Norwalk), pp 945-958.

[27] Kanner, L. (1943) "Autistic disturbances of affective contact", Nerv. Child 2. 217-250.

[28] Kasari, C., Sigman, M.D., Baugmgartner, P. and Stipek, D.J. (1993) "Pride mastery in children with autism", J. Child Psychol. Psychiat. 34, 353-362.

[29] Kemper, T.L. and Bauman, M. (1993) "The contribution of neuropathologic studies to the understanding of autism", Behav. Neurol. 11, 175-187.

[30] Kohonen, T. (1982) "Self-organized formation of topologically correct feature maps", Biol. Cybernetics 43, 59-69.

[31] Kuffler, S. (1953) "Discharge pattern and fuctional organization of mammalian retina", J. Neurophysiol. 16, 37-68.

[32] Land, R., Folstein, S.E. and Isaacs, C. (1991) "Spontaneous narrative-discourse performance of parents of autistic individuals", J. Speech Hear Res. 34, 1339-1345.

[33] Malsburg, C. (1973) "Self-organization of orientation sensitive cells in the striate cortex", Kybernetik 14, 85-100.

[34] Meltzoff, A.N. and Gopnik, A. (1993) The Role of Imitation in Understanding Persons and Developing a Theory of Mind: Perspectives from Autism (Oxford University Press, Oxford. UK).

[35] Mountcastle, V.B. (1957) "Modality and topographic properties of single neurons of cat's somatic sensory cortex", J. Neurophysiol. 20, $408-434$

[36] Piven. J., Berthier, M.L., Starkstein, S.E., Nehme, E., Pearlson, G. and Folstein, S. (1991) "Magnetic resonance imaging evidence for a detect of cerebral cortical development in autism", Am. J. Psychiat. 147, 734-739.

[37] Raymond, G., Bauman, M. and Kemper, T. (1989) "The hippocampus in autism. Golgi analysis. Programs and Abstracts", Child Neurol. Soc, 26, 483-484

[38] Real, R.A. and Imig, T.H. (1980) "Tonotopic organization in auditory cortex of the cat", J. Comp. Neuro. 192, 265-291.

[39] Ritter, H. and Schulten, K. (1989) "Convergency properties of Kohonen's topology conserving maps: fluctuations, stability and dimension selection", Biol. Cybernetics 60, 58-71.

[40] Ritvo, E. and Freeman, B. (1978) "National society for autistic children definition of the syndrome of autism", J. Autism Childhood Schizophrenia 8, 162-169.

[41] Robson, J.G. (1975) Receptive Fields: Neural Representation of the Spatial and Intensive Attributes of the Visual Image (Academic Press, New York)

[42] Rumelhar, D.E. and McClelland, J.L. (1985) Parallel Distributed Processing (MIT Press, Cambridge, MA).

[43] Rutter, M. (1978) "Diagnosis and definition of childhood autism", J. Autism Childhood Schizophrenia 8, 139-384.

[44] Shah, A. and Frith, U. (1993) "Why do autistic individuals show superior performance on the block design task?", J. Child Psychol. Psychiat. 34, 1351-1364. 
[45] Spitzer, M. (1995) "A neurocomputational approach to delusions", Compr. Psychiat. 36, 83-105.

[46] Szentagothai, J. (1967) "The module concept in cerebral cortex architecture", Brain Res. 95, 475-496.

[47] Upton, D. and Corcoran, R. (1995) "The role of the right temporal lobe in card sorting: a case study". Cortex 31, $405-409$.
[48] WHO (1993) World Health Organization: Mental Disorders: the ICD-10 Classification of Mental and Behavioural Disorders (Diagnostic Criteria for Research, Geneva, Switzerland).

[49] Wing, L. and Gould, J. (1979) "Severe impairments of social interaction and associated abnormalities in children: epidemiology and classification", J. Autism Dev. Disord. 9, 11-29. 


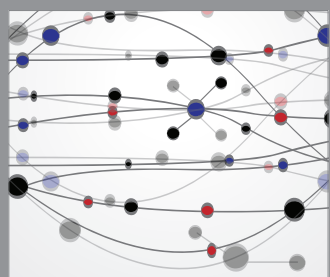

The Scientific World Journal
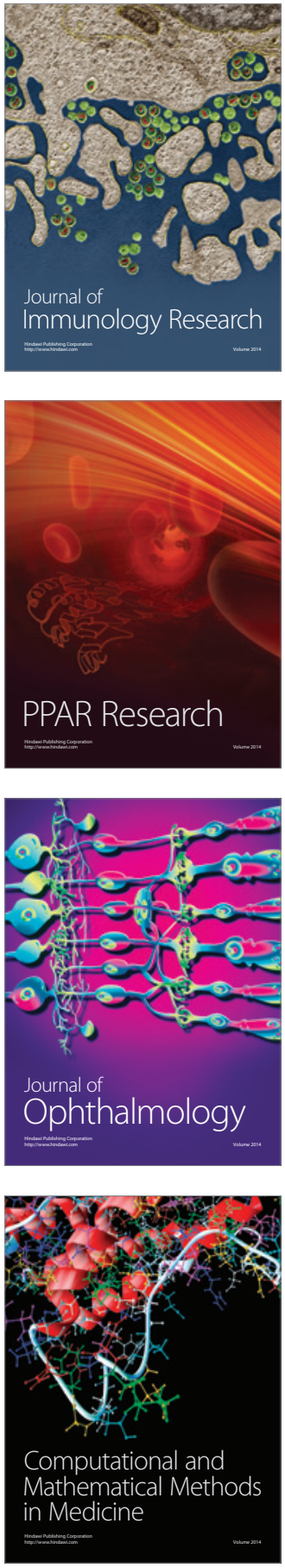

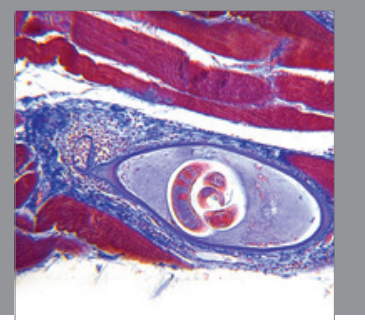

Gastroenterology

Research and Practice
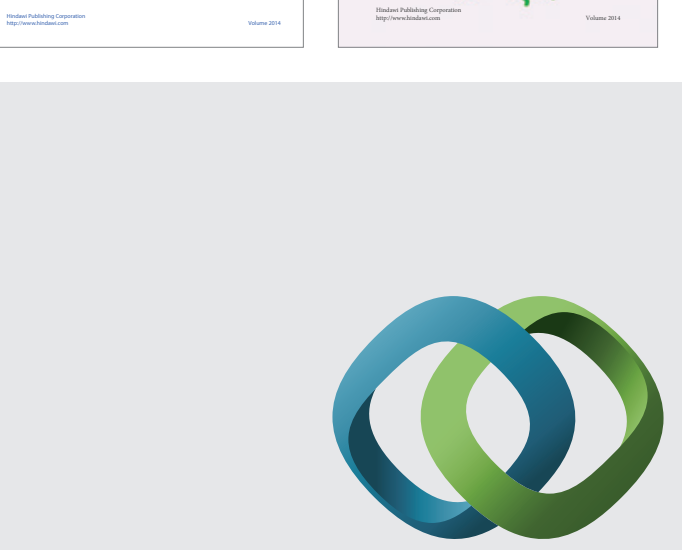

\section{Hindawi}

Submit your manuscripts at

http://www.hindawi.com
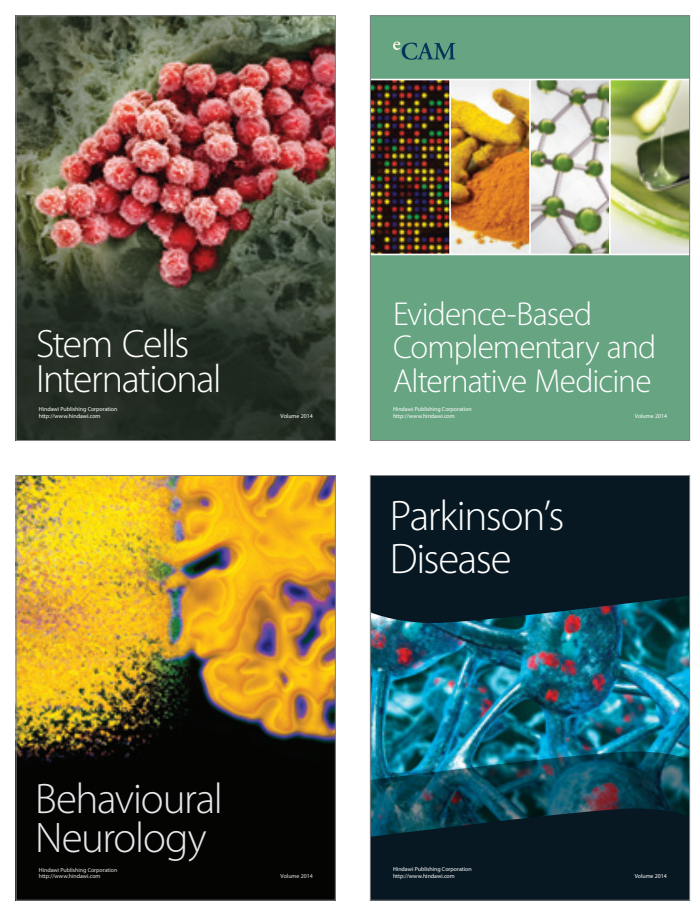

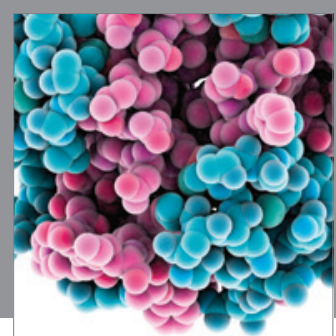

Journal of
Diabetes Research

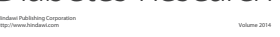

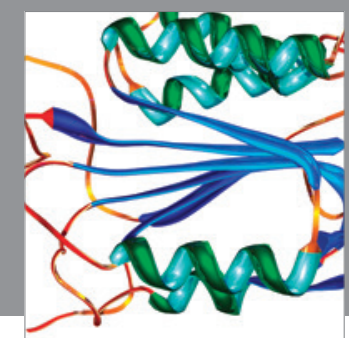

Disease Markers
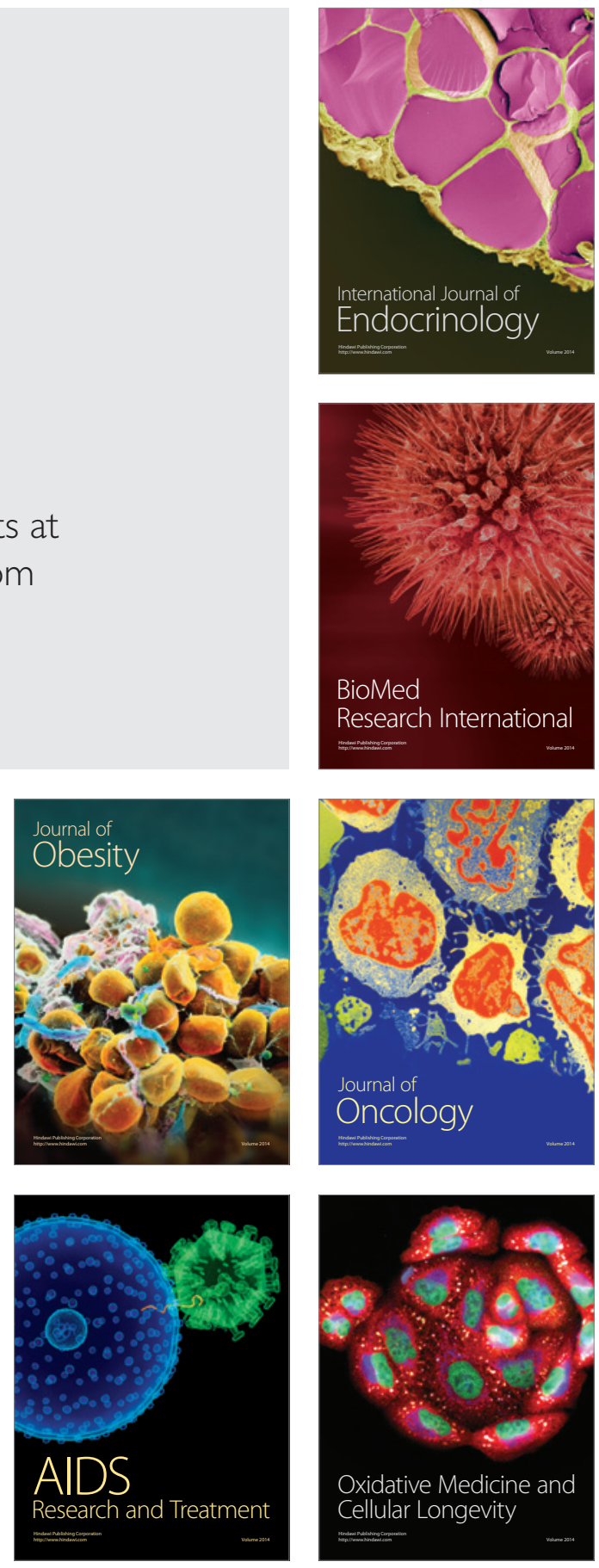